IZA DP No. 8782

Cultural Diversity: A Matter of Measurement

Peter Nijkamp

Jacques Poot

January 2015

Forschungsinstitut

zur Zukunft der Arbeit

Institute for the Study

of Labor 


\title{
Cultural Diversity: A Matter of Measurement
}

\author{
Peter Nijkamp \\ VU University Amsterdam \\ and IZA
}

Jacques Poot

NIDEA, University of Waikato

and IZA

\author{
Discussion Paper No. 8782 \\ January 2015
}

\author{
IZA \\ P.O. Box 7240 \\ 53072 Bonn \\ Germany \\ Phone: +49-228-3894-0 \\ Fax: +49-228-3894-180 \\ E-mail: iza@iza.org
}

Any opinions expressed here are those of the author(s) and not those of IZA. Research published in this series may include views on policy, but the institute itself takes no institutional policy positions. The IZA research network is committed to the IZA Guiding Principles of Research Integrity.

The Institute for the Study of Labor (IZA) in Bonn is a local and virtual international research center and a place of communication between science, politics and business. IZA is an independent nonprofit organization supported by Deutsche Post Foundation. The center is associated with the University of Bonn and offers a stimulating research environment through its international network, workshops and conferences, data service, project support, research visits and doctoral program. IZA engages in (i) original and internationally competitive research in all fields of labor economics, (ii) development of policy concepts, and (iii) dissemination of research results and concepts to the interested public.

IZA Discussion Papers often represent preliminary work and are circulated to encourage discussion. Citation of such a paper should account for its provisional character. A revised version may be available directly from the author. 


\section{ABSTRACT}

\section{Cultural Diversity: A Matter of Measurement}

Cultural diversity - in various forms - has in recent years turned into a prominent and relevant research and policy issue. There is an avalanche of studies across many disciplines that measure and analyse cultural diversity and its impacts. Based on different perspectives and features of the available data, a great variety of diversity indicators have emerged. The present paper aims to highlight some critical issues involved in applying such measures of cultural diversity. A selection of commonly used or recently advocated measures are reviewed. Measures of population diversity can be calculated at different spatial scales and used to analyse spatio-temporal heterogeneity. Additionally, there is a growing interest in measuring spatial dependence, particularly in the form of segregation or clusters. We conclude that there will be in the future considerable scope for adopting multidimensional and cultural distance-weighted measures of diversity. Such measures will be increasingly calculated by means of rich geo-referenced longitudinal micro data. However, adopted measures must be better motivated by behavioural theories. Further research on the determinants and impacts of observed measures of diversity is also likely to be fruitful, particularly in a dynamical setting.

JEL Classification: C00, D63, J15, R23, Z13

Keywords: diversity, dissimilarity measurement, ethnicity, culture, segregation, polarization, fractionalization

Corresponding author:

Jacques Poot

National Institute of Demographic and Economic Analysis

University of Waikato

Private Bag 3105

Hamilton

New Zealand

E-mail: jpoot@waikato.ac.nz

\footnotetext{
* This is a draft of Chapter 2 forthcoming in J. Bakens, P. Nijkamp and J. Poot (eds) The Economics of Cultural Diversity. (Cheltenham UK: Edward Elgar, 2015). An earlier version of this paper was presented at the Workshop E Pluribus Prosperitas: The Economics of Cultural Diversity, Tinbergen Institute, Amsterdam, April 15-17, 2013 and at a seminar at the Netherlands Interdisciplinary Demographic Institute (NIDI) in The Hague on October 29, 2014. We would like to thank Annekatrin Niebuhr, Daniel Arribas-Bel and workshop/seminar participants for comments. Ceren Ozgen, Alina Todiras and Emma van Eijndhoven provided helpful input in developing this paper. The research reported in the paper was initially conducted as part of the 2009-2013 Migrant Diversity and Regional Disparity in Europe (MIDI-REDIE) project, funded by the NORFACE-Migration research programme, www.norface-migration.org. Jacques Poot also acknowledges a grant from the Netherlands Institute for Advanced Study in the Humanities and Social Sciences (NIAS) and grant UOWX1404 provided by the New Zealand Ministry of Business, Innovation \& Employment (MBIE) for the research programme Capturing the Diversity Dividend of Aotearoa New Zealand (CaDDANZ).
} 


\section{INTRODUCTION}

Migration and cultural diversity are intertwined phenomena. Following several decades of growing numbers of immigrants in the developed world, the populations of host countries have become more diverse: culturally, socio-economically, but also spatially. This transformation has been particularly prominent in Europe. During the last three decades, the foreign-born population in Europe has increased more than in any other part of the world.

While migrants from some backgrounds adopt the dominant culture of the host society quickly, others maintain the culture of their home country and pass this on to their children and subsequent generations. Aspects of foreign cultures are also adopted by the host population and people may feel individually attached to several cultures. Moreover, cultures are never static but evolve and adjust in migrants' countries of origin and countries of destination.

The issue of cultural diversity has in recent years prompted a wealth of scientific research, both conceptually and empirically. In our 'age of migration' we observe interesting distinct patterns of living, working, communicating, bonding and other behavior among and between different cultural groups. The question whether the socio-economic and spatial outcomes of cultural diversity are positive or negative has led to a rapidly growing literature. This literature is reviewed briefly in the next section. To date, there have been many contrasting findings. These depend inter alia on: the geography and spatial scale of analysis in the countries concerned; time scale; classification of the groups considered; socio-economic conditions; institutions; and the selected definition of diversity. This prompts the questions whether, firstly, there is a best way to measure cultural diversity - non-spatially or spatially - and, secondly, whether measurement of the impacts of diversity is sensitive to the choice of a diversity indicator. These questions are the focus of the present chapter.

A vast collection of measures/indicators of diversity of populations has been proposed in the literature. While such measures can all be applied in principle to cultural diversity, many have their origin in other areas of socio-economic research or even in other disciplines. To introduce the need for a systemic perspective on measuring cultural diversity, we just mention here six - rather randomly selected studies on diversity, which use different measures of diversity among population groups. Firstly, Åslund and Skans (2009) develop an index of systematic segregation 
in which they account for covariates in a nonparametric way. Secondly, Constant and Zimmermann (2008) define an ethnosizer index which aims to measure the intensity of a person's ethnic identity. Thirdly, Fearon (2003) uses an index of ethnic fractionalization and an index of cultural fractionalization (with structural distances between languages being a proxy for cultural distance in the latter measure). Fourthly, Olfert and Partridge (2011) investigate the creative classes in cultural communities and employ a modified global-region-of-birth fractionalization index. Fifthly, Ottaviano and Peri (2006) measure the effect on wages and rents in US cities by means of the standard fractionalization index. Finally, Wong and Shaw (2011) use an exposure measure of ethnic segregation but take an activity space approach rather than the conventional residential perspective.

It is evident from the above examples that there is a wealth of indicators for measuring non-spatial and spatial diversity or heterogeneity (we will use the terms interchangeably) of populations. Following a short review of the literature on cultural diversity and its socio-economic impacts in the next section, we outline some general principles regarding measurement of diversity in Section 3. This is followed by a discussion of common measures of population heterogeneity in Section 4. The penultimate section is concerned with the combination of spatial and population heterogeneity. We conclude with some retrospective and prospective remarks in Section 6.

\section{THE SIGNIFICANCE OF CULTURAL DIVERSITY}

Socio-cultural diversity embodies a host of cultural, ethnic, religious, political and demographic factors. These are to be distinguished from economic characteristics (wealth, ability, education, occupation, etc.). Even without socio-cultural diversity, the presence of people with different socio-economic attributes and heterogeneous preferences will lead through competitive market forces and through societal institutions to a non-random 'sorting' of people. This sorting can take place spatially within cities (as already explained by urban land rent theories of Alonso, 1964, and Muth, 1971; but see also: Borjas, 1998; Ihlanfelt and Scafidi, 2002; Johnston et al., 2007), but also more broadly geographically across regions (e.g., Roback, 1982; Moretti, 2011). Finally, sorting can be by skills and occupations across and within firms (e.g., Heckman et al., 1998). 
In the economist's classic view of the world, the influence of intra- and intergroup relationships on economic processes has been largely assumed away. However, in recent years economists have started to pay increasingly attention to phenomena such as social capital (Putnam, 2000) and interdependence of preferences (Brock and Durlauf, 2001). In the broader social science literature, attention has already been paid much longer to the question whether and how interactions between distinct population groups have an influence on their behavior. Distinct population groups may be the result of specific social values, religious convictions, cultural attitudes, lifestyles, languages or traditions. Residential sorting - and in a stricter sense, residential segregation - reflects the economic and socio-cultural diversity among subgroups of the population. This observation is well articulated in the seminal studies of Sakoda (1971) and Schelling (1971) that focused the attention on the intensity of social bonds or linkages within a given household's neighborhood in a city. Households will then try to move to an urban district where the share of their friends and relatives is above a certain threshold. These studies have prompted an avalanche of spatial segregation research in cities (Bruch and Mare, 2006; Fossett, 2006a, 2006b; Benenson, 1998; Boal, 1978; Speare, 1974). Unfortunately, a solid quantitative test of these ideas that lends itself to generalization and comparative study is still missing. As a consequence, there is much speculation about the assumed drivers and effects of socio-cultural diversity in urban neighborhoods. Especially the definition of a clearly and unambiguously demarcated urban space that forms a justified unit of analysis for comparative study is lacking.

It is noteworthy that even leading researchers in the field have come up with rather contrasting conclusions on the impacts of diversity. We will illustrate this by briefly summarizing two important studies. First, Page (2007) highlights in his book The Difference the beneficial effects of diversity in a social environment, such as schools, firms and organizations. Diversity of membership of such social constellations appears to improve their functioning. Page seeks to explain the emergence of diversity benefits mainly in terms of the ability of organized social structures to employ a variety of cognitive tools based on training, experience and genes. His work and his positive conclusions on diversity are supported by a wealth of empirical material, pertaining to different countries and different social groupings. A thorough review of his study can be found in Ioannides (2010). 
An opposite conclusion can be found in a 2006 article entitled E Pluribus Unum by Putnam, in which he argues that (ethnic) diversity leads to an erosion of social capital. Diversity undermines communication between people and hence affects social solidarity, not only between distinct groups but also among group members. Consequently, diversity will negatively impact on social norms (like reciprocity, trust, worthiness, etc.). Based on a series of empirical studies he arrives at the conclusion that diversity has always a negative effect and undermines stable social networks, leading in the end to increased social isolation (the 'hunkering down' hypothesis).

Putnam (2000) highlighted the distinction between bonding and bridging in the development of social capital in migrant communities. The interaction of workers of different cultural backgrounds with the host population can increase productivity due to knowledge spillovers or other forms of positive externalities (see e.g. the sociocultural mixed embeddedness hypothesis by Kloosterman and Rath, 2001). This result is only an advantage up to a certain degree. When the variety of ethnic or cultural backgrounds is too diverse, fractionalization may imply excessive transaction costs for communication (the 'Babylon effect'; see Florax et al., 2005) and may therefore lower productivity.

On the other hand, cultural diversity among migrants may impact positively on host societies by enhancing the quality of life in cities and consumer choice options. A tolerant native population may value a multicultural society because it increases the range of available goods and services. Although cultural economics is a growing field (Blaug, 2001), the potential benefits of migration-induced cultural diversity on the arts - and culture in general - has hardly been investigated from an economic perspective.

Admittedly, diversity may be perceived as an unattractive feature if, e.g., natives recognize it as a distortion of national or local identity. They may discriminate against other ethnic groups and fear that social conflicts between different foreign nationalities may be imported into their own neighborhoods. Because of the spatial selectivity of migration, the impacts of the aforementioned mechanisms are likely to be amplified at the regional and urban level. Diversity may also influence subjective wellbeing of individuals in positive or negative ways (e.g., in terms of perceptions of neighborhood safety).

Given the growing importance of linkages between migrant source and destination countries through global diaspora, the perspectives of migrant source countries are 
also of great relevance. Migrants and culturally defined communities form various networks that have a range of impacts, such as on international trade and on the spatial clustering of migrant groups. In the presence of growing benefits of urban agglomeration, which the world is witnessing at present, the fact that migrants are often drawn from peripheral areas and drawn to large agglomerations may reinforce differences in socio-economic development between these areas (see Ozgen et al. 2010 for meta-analytic evidence).

Research on the socio-economic and spatial impact of cultural diversity is nowadays intensifying (see for overviews: Alesina and La Ferrara, 2005; Olfert and Partridge, 2011; Ratna et al., 2009). Economic studies of socio-cultural diversity tend to emphasize the positive externalities emerging from the presence and interaction of diverse population groups. However, growing socio-cultural diversity of the population may affect the host economy through many different channels. The net impact depends on the relative strength of these effects. A seminal study by Ottaviano and Peri (2006) demonstrated that US-born citizens experienced both a wage increase and a rising rental price of their house in metropolitan areas in which the share of foreign-born people increased. They conclude that there is a significant positive effect of cultural diversity on the productivity of the native born.

A promising start has also been made with the analysis of the interrelationships between cultural diversity and economic performance in Europe (Bellini et al., 2008; Suedekum et al., 2014; Ozgen et al., 2012). While most research finds that there is a positive causal link from migrant diversity to regional productivity and innovation, the relationship is complex and may be specific to certain industries, types of firm, or types of workers (for a review, see Ozgen et al., 2014). In addition, there has been a series of new studies - largely on the basis of micro data - that have aimed to assess the disaggregate benefits of cultural diversity, for instance, on the degree of firm's innovativeness (e.g., Ozgen et al., 2013), on entrepreneurship (e.g., Audretsch et al., 2010; Sahin et al., 2010), on plant productivity (e.g., Haas and Lucht, 2013; Trax et al., 2012), on labor demand (e.g., Brunow and Blien, 2014), on income (e.g., Brunow and Brenzel, 2012) but also on social issues such as trust (e.g., Gundelach and Freitag, 2014), social cohesion (e.g., Sturgis et al., 2014) and subjective wellbeing (e.g., Longhi, 2014).

The above sketched contrasting viewpoints and findings suggest that, on one hand, diversity increases contacts between diverse people and thereby improves 
mutual understanding and trust and, on the other hand, diversity will magnify conflicting interests between groups and thereby create the basis for a disintegration of society. It is clear that both sets of phenomena are potentially concurrent manifestations of reality with the relative strengths of each being context specific and probably also dependent on the operationalization of the concept of diversity.

The literature on diversity has an abundance of related concepts. Some of these are non-spatial and refer to the (multi-dimensional) composition of a given population. Others are concerned with the varying presence of population sub-groups across space. Two common concepts of the latter are segregation and (spatial) concentration. Concentration is usually interpreted as referring specifically to geography or space, i.e. the extent to which a population defined by a certain attribute is unevenly dispersed across spatial units. Segregation refers to the degree to which two or more groups defined by one attribute (age, sex, ethnicity) are distributed unequally across another attribute (location, occupation, wealth, etc.). Clearly, a relatively low segregation implies that a difference between the groups is not discernible for the given classification. For example, if there was no gender segregation across occupations, knowing a person's occupation would not be informative of the person's gender (this idea of reducing attributes to those that have predictive power in comparing individuals is the foundation of rough set theory, see Pawlak, 1997).

A wealth of conceptual and empirical studies has been undertaken to understand and map out segregation and concentration (see, e.g., Friedrichs, 1998; van Kempen, 2005; Musterd and van Kempen, 2009; Phillips, 1998). In many cases, a high level of segregation and a high spatial concentration of certain socio-cultural or ethnic groups are regarded as undesirable situations that may reinforce a disadvantaged socioeconomic position of certain population groups. This has prompted a debate on policies to reduce segregation in cities, for instance, by introducing a residential quota system, by fostering urban economic policies that would benefit less privileged people, or by implementing a system of affirmative action which would give priority to disadvantaged ethnic or socio-cultural groups in the local labor or housing market (see e.g., Feinberg, 1985; Moro and Norman, 2003).

Additionally, the policy debate in many European countries has focused on improving the skill composition of the immigrant flow. In some countries attempts are made to attract high-skilled temporary and permanent immigrants to specific 
industries suffering from labor shortages (e.g., in the IT industry). Cultural diversity of migrants has to date played only a minor role in this policy debate.

Hence, a fundamental question is whether, and to what extent, migrant diversity based on different origins and socio-economic capabilities - may be seen as a potentially positive contributor to economic welfare, and to what extent it influences socio-economic disparity. Additionally, the way in which residential choice and mobility of migrants impacts on socio-economic outcomes at specific locations is also of great interest.

All empirical studies must operationalize concepts of cultural diversity and spatial segregation of population sub-groups. Many use the same measures but rarely assess the properties and suitability of such measures in the specific context. Following a brief discussion in Section 3 of concepts, terminology and notation that can be applied to all measures of cultural diversity and segregation discussed in this chapter, we first review measures of heterogeneity of (sub) populations in Section 4 and then diversity of places in Section 5.

\section{GENERAL PRINCIPLES}

Diversity is a concept that is nowadays used in a broad array of social science research. In an edited volume by Knotter et al. (2011), the following application fields are mentioned and extensively discussed: social anthropology, sociology, criminology, ecology, linguistics, architecture, urban planning, geography, economics, management and organization, psychology, law, and public policy/political science. There are evidently different meanings and terminology (e.g. heterogeneity, diversity, dissimilarity) attached to the concept of diversity, but a critical question is: how to measure it? In socio-economic research on diversity the fractionalization index (a measure of diversity among people) and the segregation index (a measure of diversity among places) have certainly become the 'market leaders'. The former refers to the probability of two persons meeting who are not of the same type (Alesina et al., 2003). The latter refers to the proportion of people of a particular type who would have to be redistributed to make their spatial distribution (or with respect to some other type of classification, such as occupation - see Duncan and Duncan, 1955) identical to that of the rest of the population. 
At the most fundamental level, all such measures are concerned with a population (census or sample) of individuals who are classified across a range of characteristics, also referred to as attributes. In order to assign people to groups, the attributes are assumed to be qualitative/categorical variables. They may be ordinal (e.g. education) or not (e.g. ethnicity). Attributes drawn from continuous distributions can be converted into categorical variables by means of some process of discretization (e.g. from exact age to age groups). Attributes are measured at different points in time, hence the date and time of observation is itself an attribute of the person being observed. Given the importance of geographical space, the attribute of location is often considered separately from the others. Geographical space is partitioned into areas and individuals are located in one of these areas.

Once groups have been defined in terms of one or more attributes, the population can be partitioned into groups such that within a group all individuals have identical observed attributes. Even though the description of groups may be detailed (i.e. many attributes are considered), some unobserved heterogeneity may remain, which can of course influence statistical analyses if it is non-randomly related to the observed heterogeneity.

Traditionally, cultural diversity research focused only on one aspect and a corresponding classification: culture, ethnicity, race, country of birth, language, citizenship, religion, etc. However, it is clear that socio-economic impacts may depend on attributes drawn from multiple classifications. Additionally, in our increasingly mobile world with a growing complexity of migration types (e.g. Poot et al., 2008), the number of relevant attributes is increasing and may include migrant visa status, spatial selectivity and international networks. It is therefore not surprising that Vertovec (2007) coined the term 'super-diversity' to describe this new reality. On the other hand, when either the number of classifications is high and/or the categorization in each classification is rather fine, many of the potential combinations are not observed in practice. This is referred to as the 'sparse matrix' or 'empty cells' problem. Another interesting issue when considering many attributes is the extent which the measure of overall diversity is simply the sum of diversity measures for each of the attributes. Such additivity would generally require at least independence across classifications.

This general setting is identical to that of the multi-way cross tabulation of categorical data (also called a contingency table), which fundamentally refers to a 
multivariate discrete distribution (with location in our context being one of these variables). Many methods have been developed for analyzing such data, with the core objective usually being the association and interaction (or independence) between attributes (e.g., Bishop et al., 1975). Information theory has turned out to be helpful for analysis of such tables (e.g., Gokhale and Kullback, 1978) and most statistical software nowadays contains appropriate procedures, such as log-linear or general linear models. For a recent review of this literature, see Fienberg (2011). In this context, measures of diversity can be interpreted as the 'moments' of the multivariate distribution. Such measures could be concerned, for example, with the dominance of the modal group, bimodality (polarization), dispersion or evenness, skewness, etc.

For any given classification, the 'distance' between possible outcomes may be important to describe the 'intensity' of diversity. While for cardinal variables this is straightforward (the distance between age groups is the difference in mean ages), for ordinal (e.g. qualifications) and non-ordinal (e.g. language) classifications it may depend on multiple characteristics of each category. For example, a group of people equally distributed across their four native languages French, Italian, Spanish and Portuguese may be considered less diverse than an equally sized group equally distributed across French, Japanese, Russian and Chinese. Distance-weighted measures of diversity will be described later. It is useful to note that the recent emergence of 'big data' (e.g., McAfee and Brynjolfsson, 2012), such as on interactions between individuals measured through email and mobile phone traffic, or face-to-face visits monitored through transportation data, may provide new sources of information that signal socio-cultural distance between groups.

We will now introduce some notation that will be maintained throughout the remainder of the chapter. We assume that we focus on a population of size $N_{t}$ observed at time $t(t=1,2, \ldots, T)$, in which each individual has a set of attributes (characteristics) $C$ and is located in area $a(a=1,2, \ldots, A)$. A group $g(g=1,2, \ldots, G)$ is defined by people with identical attributes. The number of people who belong to group $g$ in area $a$ is denoted by $P_{\text {gat }}$. A subscript $\bullet$ denotes aggregation over that index. Hence $N_{t} \equiv P_{\text {.ot }}=\sum_{g=1}^{G} \sum_{a=1}^{A} P_{g a t}$.

We consider two types of distance: geographical distance and social-cultural distance. The geographical distance may be measured in various ways, as in spatial econometrics (e.g. Anselin, 1988). Socio-cultural distance between groups may also 
be conceptualized in different ways (e.g., Karakayali, 2009; Desmet et al., 2009). The geographical distance between areas $r$ and $s$ (defined, for example, by the distance between population centroids) is denoted by $\delta_{r s}$ and social-cultural distance between groups $i$ and $j$ by $\sigma_{i j}$. In both cases we normalize these distances to be on the interval $(0,1]$ when $r \neq s$ and $i \neq j$, but $\delta_{a a}=0$ for all $a$ and $\sigma_{g g}=0$ for all $g$. These distance measures satisfy $\delta_{r s}=\delta_{s r}$ and $\sigma_{i j}=\sigma_{j i}$ for all $r, s, i, j$; and also $\delta_{r s} \leq \delta_{r q}+\delta_{q s}$ and $\sigma_{i j} \leq$ $\sigma_{i k}+\sigma_{k j}$. Consider for example linguistic distance: $\sigma_{i j}$ would be close to zero if $i$ refers to Dutch and $j$ to Flemish, whereas $\sigma_{i j}$ would be close to one if $i$ refers to Dutch and $j$ to Japanese.

Because group membership generally refers to multiple classifications, distances between groups are multidimensional too and depend on the similarity between the categories of the classifications (e.g., Bossert et al., 2003). Even within one classification, such as language, distance can be determined by the combination of a number of criteria (Greenberg, 1956; McMahon and McMahon, 2005). The idea of quantifying cultural (power) distance was introduced by Hofstede (2001). Various subjective and objective methodologies may be used to define groups, and measure distances between groups, but this remains challenging (Shenkar, 2001). However, for the purpose of describing measures of diversity, the concepts and notation introduced in this section is adequate.

Once groups have been defined, individuals may of course transition from one group to another and from one location to another between successive points in time. This calls for a longitudinal analysis in which ${ }_{i r} \pi_{j s}(t, t+d)$ refers to the transition probability that an individual belongs to group $i$ in area $r$ at time $t$ and to group $j$ in area $s$ at time $t+d$, with $d>0$. All those who enter our observations at time 0 with given attributes $i^{*}$ and in area $r^{*}$ may be referred to as a cohort. It is clear that $0 \leq$ ${ }_{i r} \pi_{j s}(t, t+d) \leq 1$ and ${ }_{i r} \pi_{\bullet o}(t, t+d)=1$ for all $d>0$ when one group may exclusively capture attrition. If ${ }_{i r} \pi_{j s}(t, t+1)$ is independent of $t$, the transition process becomes a Markov chain.

It is plausible that transition will have some kind of 'gravity' property, as in migration and trade modelling (e.g. Anderson, 2011): ${ }_{i r} \pi_{j s}(t, t+d)$ is likely to be negatively correlated with both socio-cultural distance $\sigma_{i j}$ and geographical distance $\delta_{r s}$, while the number of transitions is likely to be positively related to $P_{i r t}$ and to $P_{j s t}$. In fact, we could gauge the extent of similarity between groups by observing actual 
transitions ('revealed preference') or by means of surveys ('subjective valuation'). Of particular interest for cultural diversity are language acquisition, cross-cultural marriage and changing cultural identity across entry cohorts (first generation migrants) and birth cohorts (higher generations). ${ }^{1}$

\section{COMMON MEASURES OF POPULATION HETEROGENEITY}

Measuring diversity is a fundamental aspect of the work of ecologists and biologists. Some of the measures that are available in the literature on biodiversity have been applied in the social sciences too; often using identical mathematical formulae but a different terminology and interpretation. Maignan et al. (2003) show that while economists are usually interested in measures of inequality with respect to quantitative phenomena, such and income or wealth, and ecologists are mostly concerned with relative and absolute abundance of different qualitative types such as species and sub-species, there is potential for a greater transfer of measures across disciplines. There is also a need for a better understanding of how different diversity measures are related to each other.

In this context, a family of measures developed by Patil and Taillie (1982) is very helpful (see also Maignan et al., 2003). Consider a group $g$ with relative frequency of occurrence $P_{g} / P_{\text {. }}$. (we drop here the area and time subscripts, $a$ and $t$ respectively, for simplicity because area and time are assumed fixed in this section). Whether we consider this group 'rare' could be signaled by a function that takes this group share to a certain power. Hence 'rarity' of group $g$ may be given by

$$
R(\beta)=\left[1-\left(P_{g} / P_{\bullet}\right)^{\beta}\right] / \beta
$$

in which we assume that $\beta \geq-1$. The value of $\beta$ determines whether we emphasize 'abundance' or 'evenness' (a small $\beta$ yields a measure of how representative the group is in the population, a large $\beta$ signals the likelihood that the group contributes to an even distribution across categories; examples follow below). Many common non-

\footnotetext{
${ }^{1}$ It is clear that the groups and transitions must be defined carefully. A migrant who becomes fluent in a host country language and who would then refer to that language as her/his 'first' language would become a member of a group that is bilingual rather than monolingual, as such a person would not, or at least not fully, 'unlearn' their native language.
} 
spatial measures of diversity are weighted averages of 'rarity' with weights given by the group shares $\left(P_{g} / P_{\bullet}\right)$ :

$$
D(\beta)=\sum_{g=1}^{G}\left(P_{g} / P_{\bullet}\right) R(\beta)=\sum_{g=1}^{G}\left(P_{g} / P_{\bullet}\right)\left[1-\left(P_{g} / P_{\bullet}\right)^{\beta}\right] / \beta
$$

We can roughly interpret $\beta$ as generating the 'moments' of the categorical distribution. The simplest measure is obtained when $\beta=-1$. It is easy to see that $D(-1)=G-1$. This is almost identical to the richness or abundance of the diversity of the population, $A B=G$, which is simply the number of distinct types. ${ }^{2}$ Of course we would expect that the larger the population we consider, the more likely it becomes that even rare groups are present. It is therefore useful to also consider indices of relative abundance such as the Margalev diversity index MA (Margalef, 1958):

$$
M A=\frac{D(-1)}{\ln P}
$$

or the Menhinick index $M E$ (e.g., Whittaker, 1977):

$$
M E=\frac{A B}{\sqrt{P_{\bullet}}}
$$

Both measures have been developed in the context of ecological diversity where sampling is common. These relative abundance measures are also useful in socioeconomic contexts where indices are compared across populations of quite different sizes.

Next, we consider the case $\beta=0$. Because $R(\beta)=\left[1-\left(P_{g} / P_{\bullet}\right)^{\beta}\right] / \beta$, it follows, using L'Hôpital's rule, that $R(0)=-\ln \left(P_{g} / P_{\bullet}\right)$ and we call the index $D(0)$ the Shannon-Weaver index $S W$ :

$$
S W=D(0)=-\sum_{g=1}^{G}\left(P_{g} / P_{\bullet}\right) \ln \left(P_{g} / P_{\bullet}\right)
$$

\footnotetext{
${ }^{2}$ Each of the 29 measures of diversity defined in this chapter will be denoted by a two letter acronym.
} 
This measure is identical to the Shannon index from information theory and is also referred to as the Shannon-Wiener or entropy index (e.g. Theil, 1972). This index can only be calculated when each group has at least one member. The index varies between zero (when there is only one type) and a maximum of $\ln G$ when all groups have an equal number of members. In order to easily compare populations that have coarse (small $G$ ) or fine (large $G$ ) classifications, we can also introduce a relative Shannon index, also referred to as the Shannon evenness index, which is $D(0) / \ln [1+D(-1)]$, i.e. which divides $D(0)$ by $\ln G$. Hence,

$$
S E=D(0) / D(-1)=-\left\{\sum_{g=1}^{G}\left(P_{g} / P_{\bullet}\right) \ln \left(P_{g} / P_{\bullet}\right)\right\} / \ln G
$$

When we consider $D(1)$, we arrive at the most commonly used measure of dispersion across categories of a classification, called the fractionalization index (also referred to as the Gini-Simpson index, see e.g. Alesina et al. 2003 and Desmet et al., 2009), which is given by

$$
F R=D(1)=\sum_{g=1}^{G}\left(P_{g} / P_{\bullet}\right)\left[1-\left(P_{g} / P_{\bullet}\right)\right]=1-\sum_{g=1}^{G}\left(\frac{P_{g}}{P_{\bullet}}\right)^{2}
$$

In economics, this index is most often used to measure (a lack of) concentration of market power among firms, in which case $P_{g}$ refers, for example, to employment or output of firm $g$ and $1-F R$ is commonly known as the Herfindahl index (HD). This index ranges between 0 (when all individuals belong to only one group) and $1-1 / G$ (when all groups have $P_{\bullet} / G$ individuals). Again, to compare fine and coarse classifications, FR can be standardized into a modified fractionalization index which varies between zero and one (see e.g. Olfert and Partridge, 2011, for an application):

$$
M F=\frac{G}{G-1} D(1)=\frac{G}{G-1}\left[1-\sum_{g=1}^{G}\left(\frac{P_{g}}{P_{\bullet}}\right)^{2}\right]
$$

Equally common in the literature is the closely related Simpson diversity index SI. This measures the probability that two randomly selected (without replacement) individuals belong to two different groups. Since this is one minus the probability that they belong to the same group, we get 


$$
S I=1-\frac{\sum_{g=1}^{G} P_{g}\left(P_{g}-1\right)}{P_{\bullet}\left(P_{\bullet}-1\right)}
$$

The last term on the right hand side is also referred to as the Simpson dominance index (DO). Greater diversity/lesser dominance implies a greater SI (smaller DO). If each group is present but has only one member, $P_{\bullet}=A B=G$ and $S I=1(D O=0)$. If everyone belongs to the same group, $S I=0(D O=1)$.

It should be noted that diversity indices such as FR and SI fail to satisfy the decomposition condition. This condition is satisfied when in a multiple classification (groups are defined by two or more attributes) the total diversity measure is equal to the sum of the diversity measures for each of the classifications, assuming that the two attributes are independently distributed across the respective categories. It is easy to see that the $S W$ index does satisfy this condition.

Another problem with the fractionalization index $F R$ and related diversity indices is that when a population consists of one large group (the 'majority') and various small groups, these diversity indices are highly correlated with the share of the large group in the population. Diversity may then be more effectively measured by calculating FR among the minorities only. Alesina et al. (2013) show that the fractionalization index $F R=D(1)$, with $g=1$ referring to the majority population, can be decomposed into

$$
F R=\sum_{g=1}^{G} \frac{P_{g}}{P_{\bullet}}\left[1-\frac{P_{g}}{P_{\bullet}}\right]=2 \frac{P_{1}}{P_{\bullet}}\left[1-\frac{P_{1}}{P_{\bullet}}\right]-\left[1-\frac{P_{1}}{P_{\bullet}}\right]^{2} \sum_{g=2}^{G} \frac{P_{g}}{P_{\bullet}-P_{1}}\left(1-\frac{P_{g}}{P_{\bullet}-P_{1}}\right)
$$

in which $2 \frac{P_{1}}{P_{\bullet}}\left[1-\frac{P_{1}}{P_{\mathbf{0}}}\right]$ can be interpreted as 'between majority and minorities' diversity and the second component as 'among the minorities' diversity. If $\frac{P_{1}}{P_{\bullet}}$ is close to one, the latter part is approximately zero and $F R$ becomes approximately a decreasing quadratic function of $\frac{P_{1}}{P_{0}}$, the share of native born. This explains the positive correlation which is observed by many researchers between the share of migrants and $F R$ (e.g., Ozgen et al. 2013).

Increasing $\beta$ further to $\beta=2$, we obtain an evenness index: 


$$
E I=D(2)=0.5 \sum_{g=1}^{G}\left(P_{g} / P_{\bullet}\right)\left[1-\left(P_{g} / P_{\bullet}\right)^{2}\right]
$$

which approaches zero when virtually everyone belongs to one group and 0.5 when all groups have equal shares. The literature usually focuses on the opposite of evenness, which is referred to as polarization. An obvious measure in terms of $D(2)$ would be simply

$$
0.5-D(2)=0.5 \sum_{g=1}^{G}\left(P_{g} / P_{\bullet}\right)^{3}
$$

(similar to the third moment that measures skewness in a continuous distribution). However, a more commonly used way of measuring polarization has been introduced by Reynal-Querol (2002):

$$
R Q=1-4 \sum_{g=1}^{G}\left(0.5-P_{g} / P_{\bullet}\right)^{2}\left(P_{g} / P_{\bullet}\right)
$$

which can also be expressed as

$$
R Q=4 \sum_{g=1}^{G}\left(\frac{P_{g}}{P_{\bullet}}\right)^{2}\left(1-\frac{P_{g}}{P_{\bullet}}\right)
$$

Such polarization measures are in practice often generalized to take account of social distances between groups (see also later in this section) and have been applied, for example, to the impact of ethnic divisions on conflict (Esteban et al., 2012).

Rather than just summarizing the 'moments' of the population distribution across the classification as we have done above, we can of course depict the unevenness of dispersion graphically by means of the Lorenz curve. This curve is obtained by ranking the groups from the rarest to the most common, and then plotting the population share of the $k$ smallest groups $(k=1,2, \ldots, G)$, which is $\sum_{g=1}^{k} P_{g} / P_{\bullet}$, on the vertical axis against $k / G$ on the horizontal axis.

An example is given in Figure 1. The extent of diversity and polarization can be gauged from the shape of the Lorenz curve. When all groups are equally represented, i.e. there is maximum diversity, the Lorenz curve is the diagonal B. A common summary measure of inequality between groups (i.e. a lack of diversity in the present 
context) is the Gini index (e.g. Rousseau et al., 1999). This index is calculated by measuring two times the area between curve A and line B.

Figure 1 about here

The Gini coefficient can be easily calculated by sorting the $G$ population shares $\left(\frac{P_{1}}{P_{\mathbf{0}}}, \frac{P_{2}}{P_{\mathbf{0}}}, \ldots, \frac{P_{G}}{P_{\mathbf{0}}}\right)$ from the smallest to the largest. Let $\left(\frac{\tilde{P}_{1}}{P_{\mathbf{0}}}, \frac{\tilde{P}_{2}}{P_{\mathbf{0}}}, \ldots, \frac{\tilde{P}_{G}}{P_{\mathbf{0}}}\right)$ denote the ranked sequence, with $\frac{\tilde{P}_{r}}{P_{\bullet}}$ the $r^{\text {th }}$ smallest fraction. Define $Y_{r}$ as the cumulative sum of the fractions up to $r$; and $Y_{0} \equiv 0$. Given this notation, the Gini coefficient of population diversity can be calculated for the population in any given area (or for all areas combined) as

$$
G A=1-\frac{1}{G} \sum_{r=1}^{G}\left(Y_{r}+Y_{r-1}\right)
$$

The Gini coefficient ranges from 0 to 1 . Its value is 0 when each group is of equal size (maximum diversity) and 1 when the entire population belongs to one group only $\left(Y_{1}=0\right)$. One benefit of the Gini coefficient is that it is a population scale-independent measure. Another benefit is that it permits a visual comparison of changes in unevenness over time or across areas. It should be noted that a given value of the Gini coefficient can represent quite different distributions. Moreover, the value of the index is influenced by granularity of the group classification, i.e. how many groups are being considered.

A related index is the Hoover index $H O$ (also referred to as the Robin Hood index) which calculates the proportion of the population of each group that would have to be redistributed in order to achieve an even distribution, with each group having $P . / G$ members. The Hoover index is given by

$$
H O=0.5 \sum_{g=1}^{G}\left|\frac{P_{g}}{P_{\bullet}}-\frac{1}{G}\right|
$$

The Hoover index is a special case of the Duncan and Duncan dissimilarity index $D I$ in which the distribution of individuals across a classification is compared for two 
populations (Duncan and Duncan, 1955). Section 4 discusses this index when the classification refers to spatial areas.

All diversity measures discussed above do not take the cultural distance between groups into account. Effectively they assume that cultural distance is at a maximum for every combination of different types $(i, j)$, i.e. $\sigma_{i j}=1$. Because many of the formulae above are weighted 'rarity' measures, it is straightforward to generalize these to modify the weighting based on how culturally dissimilar groups are. This is nicely demonstrated by Desmet et al. (2009) on the basis of a family of measures of social effective antagonism, introduced by Esteban and Ray (1994). Following these authors, the aggregate level of social effective antagonism can be defined as

$$
K(\gamma, \Sigma)=\sum_{g=1}^{G} \sum_{h=1}^{G} \frac{P_{g}}{P_{\bullet}}\left(\frac{P_{h}}{P_{\bullet}}\right)^{1+\gamma} \sigma_{g h}
$$

in which $\Sigma$ is the cultural distance matrix and $\gamma$ is a parameter that 'tunes' the measure with respect to abundance or evenness. Desmet et al. (2009) note that if $\gamma=0$ and $\boldsymbol{\Sigma}_{\max }$ is a matrix with $\sigma_{g h}=1$ for all $g \neq h$ and $\sigma_{g g}=0$ for all $g$, then $K\left(0, \boldsymbol{\Sigma}_{\max }\right)$ is the fractionalization index FR of Eq. (7). For intermediate cases, with $0<\sigma_{g h}<1$ for some $g \neq h, D F$ is the cultural distance-based fractionalization index which effectively measures the population-weighted average cultural distance: ${ }^{3}$

$$
D F=K(0, \Sigma)=\sum_{g=1}^{G} \sum_{h=1}^{G} \frac{P_{g}}{P_{\bullet}} \frac{P_{h}}{P_{\bullet}} \sigma_{g h}
$$

Similarly, $K\left(1, \boldsymbol{\Sigma}_{\max }\right)$ can be shown to be identical to the Reynal-Querol polarization index $R Q$ given in Eqs. (13) and (14), while the polarization equivalent in the general case becomes the one of Esteban and Ray (1994):

$$
E R=K(1, \Sigma)=\sum_{g=1}^{G} \sum_{h=1}^{G} \frac{P_{g}}{P_{\bullet}}\left(\frac{P_{h}}{P_{\bullet}}\right)^{2} \sigma_{g h}
$$

\footnotetext{
${ }^{3}$ Desmet et al. (2009) also discuss a special case in which there is a large cultural distance between the 'host population', which tends to be the majority, and minority groups - but not between the minority groups themselves.
} 
Rather than pre-allocating individuals to groups in terms of several characteristics, the analysis of cultural-distance weighted diversity can also be conducted at the micro level of individual data, see Bossert et al. (2011). All measures described in this section can be calculated at any spatial scale and the values of any given diversity index can be compared for areas at any given spatial scale. Alternatively, we could fix the group and consider how diverse or polarized a given spatial distribution of a given population group $g$ is by calculating measures that simply swap area and group subscripts, and run sums over $A$ areas rather than $G$ groups in the above formulae. However, in practice we often want to focus on the diversity of among groups and places simultaneously. The next section therefore reviews a range of global and local measures of cultural diversity among places.

\section{DIFFERENCES IN POPULATION HETEROGENEITY BETWEEN PLACES}

In this section we introduce several statistics that can be used to capture spatial patterns of diversity. In all equations that follow we will again use a common notation. Some of the spatial measures are referred to as global measures in that they provide a summary measure of the spatial pattern across all areas, whereas others are local measures in that they are calculated for each area. ${ }^{4}$ Let $P_{\text {gat }}$ again refer to the population of group $g(=1,2, \ldots, G)$ in area $a(=1,2, \ldots, A)$ at time $t(=1,2, \ldots, T)$. A subscript • refers again to the sum over that particular subscript ( $t$ is removed when the analysis is purely cross-sectional). Each area $a$ has a set of neighborhood areas/spaces $S$ (to be defined in detail later) that are indexed by $s_{a}$ and numbered from 1 to $S_{a}$.

Massey and Denton (1988) refer to the extent to which the spatial dispersion of a particular population group is different from the spatial dispersion of the general population as segregation. They classify 20 commonly used measures of segregation in terms of five distinct features (evenness, exposure, concentration, centralization and clustering) of the spatial distribution of minorities that such measures can potentially convey. They calculate all 20 measures with data on the location of Hispanics, blacks, Asians and non-Hispanic whites (the latter were defined as the

\footnotetext{
${ }^{4}$ Assuming the availability of geo-referenced data, all of the diversity measures reviewed in the previous section can be calculated for any given area. However, in this section we are interested in how such a diversity measure of an area compares with those of other areas. Local spatial measures of diversity can then refer to, for example, the average diversity of areas surrounding any given area.
} 
majority population) in US metropolitan areas. They use factor analysis to identify the distinct types of information these measures convey and the measures that had the greatest factor loadings. Their conclusion is that their five posited features of segregation can all be, at least to some extent, identified in the data and that some of the 20 segregation measures capture these features better than others. Hence for each feature they identify a measure that signals this feature best in the data (based on the factor analysis and other information).

Two of the preferred five measures do not take the geography of population distribution explicitly into account. One of these, the dissimilarity index, informs on evenness. The other is concerned with exposure of a minority group, in terms of potential contact, either with members of the own group (the isolation index) or members of the majority group (the interaction index). These indices will be described mathematically below.

Three features of segregation that take geography explicitly into account are concentration, centralization and clustering. Concentration measures are concerned with population density and quantify the relative amount of physical space occupied by a given group. Massey and Denton (1988) define a relative concentration index that describes this feature of spatial dispersion in the best way. Centralization refers to the extent to which a given group is located near the center of a city or region, e.g. the Central Business District or the largest city respectively. An absolute centralization index is Massey and Denton’s preferred measure of centralization. Finally, clustering refers to the extent to which the distribution of a given group is in a 'contiguous and closely packed' way, thereby creating enclaves. For this feature Massey and Denton (1988) recommend a spatial proximity index. However, the huge growth in spatiallyreferenced data and Geographic Information Systems (GIS) since the 1980s has led to many new developments in the spatial statistics and spatial econometrics literatures that can also applied to measuring diversity. The most common measures of this type will be reviewed later in this section.

One of the most common global, i.e. 'averaged' across areas, spatial diversity measures cited in the literature is the dissimilarity index, which - as just noted above - is advocated by Massey and Denton (1988) as the best measure of spatial (un)evenness (when not geo-referenced). The index is a measure of displacement the proportion of people in group one which would have to relocate in order to make their distribution identical to that of group two (Duncan and Duncan, 1955). When the 
dissimilarity index is computed for one group (a minority) and the remainder of the population combined, it is known as the segregation index. The group segregation index for group $g$ across area units $a(=1,2, \ldots, A)$ is ${ }^{5}$

$$
D I_{g}=\frac{1}{2} \sum_{a=1}^{A}\left|\frac{P_{g a}}{P_{g \bullet}}-\frac{\left(P_{\bullet a}-P_{g a}\right)}{\left(P_{\bullet \bullet}-P_{g \bullet}\right)}\right|
$$

Note that, while being a global index, the group segregation index provides very limited information on clustering patterns and will only reveal an average situation for the group. It does not take account of the location of group clusters, a phenomenon known as the checkerboard problem (Brown and Chung, 2006). The checkerboard problem recognizes that there may be one big cluster of a group, or many small communities scattered around the total area, but no way of knowing which one is present from a global index calculation. The Moran's I index of spatial correlation provides a global spatial measure that informs on which of these patterns is more plausible (see below).

The segregation index $D I_{g}$ can be interpreted as the fraction of the group $g$ 's population that would have to be redistributed in order for the spatial distribution of group $g$ to become the same as that of the rest of the population. Such redistribution could lead to unrealistic changes in the population of various areas. In some contexts it is more meaningful to calculate a modified index that measures the fraction of group $g$ and the fraction of the rest of the population that would need to be both redistributed to achieve identical spatial distributions, under the condition that the area populations remain constant. Such an index was earlier applied by Van Mourik et al. (1989) to the case of occupational segregation, where the standard segregation index measures the percentage of women who would need to change occupation to equalize the male and female distribution of the labor force across occupations. Any such redistribution is likely to imply unrealistic changes in the total number of people in various occupations. The latter may be assumed to be demand determined. Van Mourik et al.'s modified segregation index calculates the percentage of both women and men that would need to change occupation to have an equal distribution across

\footnotetext{
${ }^{5}$ Not that for simplicity we did not use the subscript $a$ for each of the diversity measures in the previous section, even though they can be calculated for each area $a$. In this section we will consider some measures that are specific to a group $g$ and to an area $a$, while others are 'averaged' across groups or across areas. Subscripts for the diversity measures are therefore useful from hereon.
} 
genders and unchanged totals in each occupation. The equivalent situation in spatial segregation is to consider, for example, relocating migrant and native born households in a social housing program such that their spatial distribution is equalized and the housing stock in each area remains unchanged. The modified segregation index is

$$
V M_{g}=\frac{1}{2 P_{\bullet \bullet}} \sum_{a=1}^{A}\left(\left|P_{g a}-P_{g \bullet} \cdot \frac{P_{\bullet a}}{P_{\bullet \bullet}}\right|+\left|\left(P_{\bullet a}-P_{g a}\right)-\left(P_{\bullet \bullet}-P_{g \bullet}\right) \frac{P_{\bullet a}}{P_{\bullet \bullet}}\right|\right)
$$

The modified segregation index turns out to be equal to the conventional segregation index times a factor that depends on the fraction of the total population that is in group $g$ (for the proof, see Van Mourik et al. 1989):

$$
V M_{g}=2 \frac{P_{g \bullet}}{P_{\bullet \bullet}}\left(1-\frac{P_{g \bullet}}{P_{\bullet \bullet}}\right) D I_{g}
$$

The isolation index is Massey and Denton's (1988) preferred measure of the degree of potential exposure of individuals to members of their own group (isolation) or another group (interaction). The isolation index captures the extent to which members of a population group are disproportionately located in the same areas, i.e. they are more clustered. Consider first the weighted average fraction of the population across all areas that belongs to group $g, \sum_{a=1}^{A} \omega_{g a} \frac{P_{g a}}{P_{\bullet a}}$, with $\omega_{g a}=\frac{P_{g a}}{P_{g \bullet}}$ and therefore $\sum_{a=1}^{A} \omega_{g a}=1$ for all $g$ (we can also measure the weighted average exposure of group $g$ to group $h$ : $\left.\sum_{a=1}^{A} \omega_{g a} \frac{P_{h a}}{P_{\bullet a}}\right)$. The isolation index $I I_{g}$ simply normalizes this average fraction in the following way (Cutler et al. 1999):

$$
I I_{g}=\frac{\sum_{a=1 \frac{\omega_{g a} \frac{P g a}{P_{\bullet a}}}{P g} P_{\bullet \bullet}}^{A} P_{g} /_{P_{\bullet \bullet}}}{1-P_{g \bullet} / P_{\bullet \bullet}}
$$

This measure captures the degree to which group members live in areas in which they are over-represented. An isolation index value of 0 indicates that the group is distributed in proportion to the total population, while a value of 1 can be interpreted as total isolation whereby all of the group locate in one or several particular areas $a$, and no-one of the rest of the population locate in those areas. Exposure can be a 
useful concept in studying acculturation. Exposure measures are usually based on information linked to a person's usual residential address, but exposure at work can be calculated when workplace addresses are known.

Another way of examining an uneven distribution of a group across a number of areas is by means of the Lorenz curve and the Gini coefficient, which were already introduced in the previous section in terms of gauging the diversity of groups in a particular area (see again Figure 1). In the spatial context a Lorenz curve is constructed by first calculating a group's fraction of the population of each area and then ranking areas from the one with the smallest fraction to the one with the largest fraction. Next, consider for each ranked area the points representing the cumulative percentage of total population up to that area on the horizontal axis and the cumulative percentage of the group's population up to that area on the vertical axis. If a group is spatially distributed identically to the total population, the Lorenz curve would coincide with the 45 degree line. Half the total area between the observed Lorenz curve and the 45 degree line is the Gini coefficient of a group's spatial segregation.

As in the case of diversity among groups, the Gini coefficient can also be easily calculated for spatial diversity. First, sort the population shares $\left(\frac{P_{g 1}}{P_{\bullet 1}}, \frac{P_{g 2}}{P_{\bullet 2}}, \ldots, \frac{P_{g A}}{P_{\bullet A}}\right)$ of group $g$ in the various regions from the smallest to the largest. For the $r^{\text {th }}$ smallest fraction, let $X_{r}$ be the cumulative sum of the corresponding shares of the regions' populations in the total population, i.e. $X_{r}=\sum_{i=1}^{r} \frac{\widetilde{P}_{\bullet i}}{P_{\bullet \bullet}}$. Similarly, $Y_{g r}$ is the cumulative sum of the corresponding shares of group $g$ 's population, $Y_{g r}=\sum_{i=1}^{r} \frac{\widetilde{P}_{g i}}{P_{g}}$ and $X_{0} \equiv 0$, $Y_{g 0} \equiv 0$. Given this notation, the Gini coefficient of segregation of group $g$ can be calculated as

$$
G G_{g}=1-\sum_{r=1}^{G}\left(X_{r}-X_{r-1}\right)\left(Y_{g r}+Y_{g, r-1}\right)
$$

The final two global non-GIS segregation or clustering measures that we consider are the closely-related Ellison and Glaeser (1997) and Maurel and Sédillot (1999) concentration indices, denoted $E G_{g}$ and $M S_{g}$ respectively. Both are derived as the correlation between location decisions made by members of a particular group, which can be positive or negative. The measures were originally derived to capture 
the geographic concentration of industries that take into account differences across industries in the firm size structure. We report here formulae proposed by Maré et al. (2012). ${ }^{6}$ A value of close to zero for either of these indices would indicate a lack of spatial segregation. The two indices differ only slightly. The $E G_{g}$ index has a more positive value for groups that are concentrated in areas with higher shares of the overall population.

Using the same notation as before, the $E G_{g}$ index is given by

$$
E G_{g}=\frac{\frac{\left\{\sum_{a=1}^{A}\left(\frac{P g a}{P g \bullet}-\frac{P_{\bullet a}}{P \bullet \bullet}\right)^{2}\right\}}{\left(1-\sum_{a=1}^{A}\left(\frac{P \bullet a}{P_{\bullet \bullet}}\right)^{2}\right)}-\frac{1}{P g \bullet}}{\left(1-\frac{1}{P g \bullet}\right)}
$$

while the $M S_{g}$ index is given by

$$
M S_{g}=\frac{\frac{\left\{\sum_{a=1}^{A}\left(\frac{P g a}{P_{\bullet \bullet}}\right)^{2}-\sum_{a=1}^{A}\left(\frac{P_{\bullet a}}{P_{\bullet \bullet}}\right)^{2}\right\}}{\left(1-\sum_{a=1}^{A}\left(\frac{P_{\bullet a}}{P_{\bullet \bullet}}\right)^{2}\right)}-\frac{1}{P g \bullet}}{\left(1-\frac{1}{P_{g} \bullet}\right)}
$$

Ellison and Glaeser (1997) suggest that in order to determine a benchmark for their measure of industry concentration, the index could be calculated for an industry which would not be considered concentrated. In our present context of spatial segregation, the spatial distributions of males and females may be used as a point of comparison. They are expected to be very similar. Hence, for both genders $E G_{g}$ (and $M S_{g}$ ) will have low values which can be used as a benchmark for gauging spatial segregation of other groups.

With respect to local diversity measures it should be noted that all of the nonspatial measures defined in Section 3 can be calculated for all areas for which data are available. Hence each index given in the previous section can be calculated for any area $a$ or any amalgamation of areas. For example, the fractionalization index for each area $a$ becomes

\footnotetext{
${ }^{6}$ Our equations differ slightly from the original formulations to reflect the focus on people rather than firms. Unlike firms, which differ in size, all people carry equal weight. Hence the final term in both the numerator and denominator of the formulae, which is in the firm case a Herfindahl index of firm concentration, simply becomes $1 / P_{g}$, as in the unweighted index of Maurel and Sédillot (1999).
} 


$$
F R_{a}=1-\sum_{g=1}^{G}\left(\frac{P_{g a}}{P_{\bullet a}}\right)^{2}
$$

The values of $F R_{a}$ can be mapped with GIS software to gauge the spatial patterns. Corresponding measures of spatial statistics such as spatial autocorrelation can also be calculated.

A simple measure of local concentration or clustering of group $g$ in area $a$ is the location quotient, also referred to as the local concentration ratio:

$$
L Q_{g a}=\frac{P_{g a} /_{\bullet a}}{P_{g \bullet} P_{\bullet \bullet}}=\frac{P_{g a} / P_{g \bullet}}{P_{\bullet a} / P_{\bullet \bullet}}
$$

$L Q_{g a}$ measures whether group $g$ 's share of the population in area $a$ is larger or smaller than group $g$ 's share of the total population (implying $L Q_{g a}>1$ and $L Q_{g a}<$ 1 respectively). Alternatively, $L Q_{g a}$ measures whether the proportion of $g$ 's population that is located in $a$ is larger or smaller than the proportion of the total population that is located in $a$.

All measures of clustering reviewed above do not account for the topological relationship of neighborhoods or areas to one another. Hence high spatial concentration may or may not coincide with spatial clustering, which is the checkerboard problem referred to earlier. This is illustrated in Figure Consider a minority distributed across areas with three population shares: high (black square), medium (grey square) and low (white square). The segregation measures discussed above will have higher values for the left checkerboards than for the right ones, but identical values for the upper and corresponding lower checkerboards.

Moran's $I$ is a global measure of clustering that yields greater values for the upper checkerboards than for the lower checkerboards. The index is a measure of spatial autocorrelation which essentially determines whether or not spatial dispersion is random. Hence Moran's I complements the global measures introduced above as the latter provide an aggregate measure of dispersion of a group across areas without a spatial reference frame, whereas Moran's $I$ calculates the degree of clustering that takes into account neighborhoods. In this context, the neighborhood of an area is 
defined as the set of areas that are within a pre-defined distance of the area considered.

Figure 2 about here

Moran's I index of the degree of global clustering of group $g$ is defined as:

$$
M I_{g}=\sum_{a=1}^{A} \frac{\left(\frac{P g a}{P g \bullet}-\frac{1}{A}\right)\left(\sum_{S_{a}=1}^{S_{a}} w_{a s_{a}}\left(\frac{P_{g s_{a}}}{P_{g} \bullet}-\frac{1}{A}\right)\right)}{\sum_{a=1}^{A}\left(\frac{P g a}{P_{g} \bullet}-\frac{1}{A}\right)^{2}}
$$

where $a$ refers to areas as before and $s_{a}$ to the areas in the neighborhood of $a$ (there are $S_{a}$ such areas). The spatial proximity weights $w_{a s_{a}}$ can be defined in various ways. For example, they can be defined as the reciprocal of the distance between the population centroid of area $a$ and the centroids of surrounding areas $s_{a}$, $\delta_{a s_{a}}$. Alternatively they can be defined simply by adjacency/contiguity (with a 'proximity' of ' 1 ' assigned to pairs of adjacent regions and ' 0 ' to non-adjacent ones). ${ }^{7}$ In all cases, the weights are row-standardized, such that $\sum_{s_{a}} w_{a s_{a}}=1$ for all $a$. One set of weights that automatically satisfies row-standardization is a set of populationbased weights, whereby the weight of neighborhood area $s_{a}$ of area $a$ is assumed to be equal to $P_{\bullet s_{a}} / \sum_{j=1}^{S_{a}} P_{\bullet j}$ if the selected area and a neighborhood area are adjacent, or if the neighborhood area is within some distance band of the selected area, and zero if otherwise.

$M I_{g}$ can be easily visualized as the slope of a regression line of the spatially weighted value of the fraction of the group's population in any area in the neighborhood of a selected area on the value of the fraction of the group's population in the selected area itself. A plot of the points through which the regression line can be drawn is referred to as a Moran scatterplot. $M I_{g}$ lies between -1 and 1 and provides a measure of how similar an area's share of the total population of group $g$ is to the population-weighted share in surrounding areas. A negative Moran's I may be indicative of isolated enclaves in which areas with a large share of a group are

\footnotetext{
7 The emergence of 'big data' on actual face-to-face encounters or online interactions through email or social media provides new and promising sources of measuring proximity between individuals and groups across areas. This can lead to new ways of measuring geographic weights matrices.
} 
surrounded by areas in which a low share of the same group can be found. This is referred to as negative spatial autocorrelation. ${ }^{8}$ A value of Moran's $I$ close to 1 is indicative of highly significant positive spatial autocorrelation that in our context can be interpreted as evidence of extensive segregation that straddles many areas.

Moran's $I$ as calculated in Eq. (29) provides only an average indication of clustering of a given group $g$. There may be a range of different spatial patterns occurring for different groups, despite similar spatial autocorrelation results. To investigate the spatial patterns of concentration, Getis and Ord's (1992) $G^{*}$ local measure of concentration can be calculated for every area $a$ and every group $g$. $G_{g a}^{*}$ is a calculation which identifies areas of neighborhood clustering that are significantly different from the average situation in the total study area (Johnston et al., 2009). Using a row-standardized spatial weights matrix $\mathbf{W}^{*}, G_{g a}^{*}$ can be calculated as:

$$
G_{g a}^{*}=\frac{\sum_{s_{a}=1}^{S_{a}} w_{a s_{a}}^{*}\left(\frac{P_{g s_{a}}}{P_{\bullet s_{a}}}-M_{g}\right)}{\sqrt{\left(\sum_{b=1}^{A} \frac{\left(\frac{P_{g b}}{P{ }^{\circ}}\right)^{2}}{A}\right)-M_{g}^{2}} \sqrt{\frac{\left(A \sum_{S_{a}=1}^{\left.S_{a} w_{S_{a}}^{*}-1\right)}\right.}{(A-1)}}}
$$

where $M_{g}=\overline{P_{g a} / P_{. a}}$ refers to the mean group share of group $g$ across all areas $a$ (see, e.g., Maré et al., 2012). Here $s_{a}$ includes the area $a$ itself in the spatial weights that are in Eq. (30) indicated by an asterisk. The index values are normally distributed $z$ scores under the null hypothesis of no spatial clustering. A value of $G_{g a}^{*}$ for an area that is greater than 1.96 indicates that there is less than a 2.5 percent chance that the high degree of concentration of group $g$ that is observed in and around the area $a$ would be observed under random location decisions (and, similarly, a value of $G_{g a}^{*}$ for an area that is less than -1.96 indicates that there is less than a 2.5 percent chance that the extreme absence of group $g$ in and near $a$ would be observed under random location decisions). $G^{*}$ values can be displayed on a map for each group to show specific neighborhoods where groups are over and under-represented.

\footnotetext{
${ }^{8}$ The question of whether the observed spatial pattern is different from a random spatial allocation of population (i.e. Moran's $I$ is statistically significant) is more complex. Two sets of standard errors can be calculated under the assumptions of standardisation and normality (Cliff and Ord, 1981; Pisatio, 2001).
} 
Various other local measures can be calculated as well. Anselin (1995) notes that Moran's $I$ is the aggregate across all areas of what he refers to as a local indicator of spatial association (LISA). Given Eq. (29), we define the local indicator $L I_{g a}$ by

$$
L I_{g a}=\frac{\left(\frac{P g a}{P g \bullet}-\frac{1}{A}\right)\left(\sum_{s_{a}=1}^{S_{a}} w_{a s_{a}}\left(\frac{P_{g s_{a}}}{P_{g} \bullet}-\frac{1}{A}\right)\right)}{\sum_{a=1}^{A}\left(\frac{P g a}{P_{g} \bullet}-\frac{1}{A}\right)^{2}}
$$

and, hence, $M I_{g}=\sum_{a=1}^{A} L I_{g a}$. As is the case with other local measures, $L I_{g a}$ can be plotted on a map; for example to identify 'hot spots': areas where high values of the index are strongly clustered (e.g. Anselin, 1995).

All spatial measures discussed in this section either refer to spatial segregation of a specific group $g$ (either dispersed or clustered across areas) or refer to the local diversity in area $a$ (either homogeneous/uniform or heterogeneous/diverse). Effectively this involves calculating indices for a column or row respectively of the $A$ x $G$ two-way cross tabulation of observed occurrences. This is depicted in Figure 3.

Figure 3 about here

Additionally, we may want to consider all cell frequencies in the table simultaneously and account, or not, for the geography that defines the various areas. For example, we could calculate the isolation index $I I_{g}$ for each group $g$ in a metropolitan area and, next, calculate a group size-weighted average of the $I I_{g}$ indices to obtain an indicator of the overall extent to which groups are isolated or spatially mixed across the metropolitan area. But there are of course many other ways of combining segregation measures for individual groups. Reardon and Firebaugh (2002) suggest six multigroup segregation indices: a dissimilarity index, a Gini index, an information theory index, a squared coefficient of variation index, a relative diversity index and a normalized exposure index. Reardon and Firebaugh (2002) list seven criteria for evaluating multigroup measures of segregation. ${ }^{9}$ They conclude that the information theory-based index, which was originally proposed by Theil and Finezza

9 These criteria are: (1) organizational equivalence; (2) size invariance; (3) transfers; (4) exchanges; (5) composition invariance; (6) additive organizational decomposability; and (7) additive group decomposability. Space constraints preclude us from elaborating on these here. See Reardon and Firebaugh (2002) for details. 
(1971) and Theil (1972), is the most satisfactory index in terms of their criteria. This index is the only one that satisfies the principle of transfers (when a person is moved from an area $i$, where its share of the population is larger than in an area $j$, to that area $j$, then segregation is reduced). Moreover, this is the only multigroup index that can be decomposed into a sum of between- and within-group components. ${ }^{10}$ The Theil index $\mathrm{TH}$ is given by the following formula:

$$
T H=\sum_{g=1}^{G} \sum_{a=1}^{A} \frac{P_{\bullet a}}{P_{\bullet \bullet}}\left[\frac{\frac{P g a}{P_{\bullet a}}\left(\ln \left(P_{g \bullet} / P_{\bullet \bullet}\right)-\ln \left(P_{g a} / P_{\bullet a}\right)\right)}{\sum_{g=1}^{G}\left(P_{g_{\bullet}} / P_{\bullet \bullet}\right) \ln \left(P_{g_{\bullet}} / P_{\bullet \bullet}\right)}\right]
$$

However, multigroup segregation measures such as $T H$ do not account explicitly for geography. A simple way of calculating an average of geographical clustering could be obtained by calculating Moran's $I$ index $M I_{g}$ for every group $g$ in a metropolitan area and then by assigning a global measure of multi-group clustering to the metropolitan area by calculating global clustering as a group size-weighted average of these indices.

Alternative measures of spatial segregation are reviewed and evaluated by Reardon and O’Sullivan (2004). These measures take account of the coordinates of the locations of individuals in the region and the proximity between individuals. This is an important extension of the various indices discussed so far which all assumed fixed boundaries of subareas $a=1,2, . ., A$ of the region or city. Distance measures, such as used in calculating the Moran's $I$ index are sensitive to the drawing of boundaries: two points that are a fixed physical distance apart may either be considered to be in the same neighborhood (i.e. the distance is zero) or in different neighborhoods (at some positive distance), dependent on where the boundary is drawn. This is referred to as the modifiable areal unit problem (MAUP). This problem and the checkerboard problem mentioned earlier in the chapter are entirely due to the defining of subareas and vanish once we adopt a fully disaggregated approach that is based on micro data on individuals and their locations.

\footnotetext{
${ }^{10}$ For example, a decomposable index of segregation of all ethnic groups should be the sum of the index of between-supergroups segregation (e.g., Africans, Asians, Europeans, etc.) and the aggregate of ethnic segregation measures for ethnicities within supergroups (e.g., Chinese, Korean, Japanese, etc. among the Asians).
} 
After identifying eight desirable properties of spatial segregation measures, ${ }^{11}$ Reardon and O'Sullivan (2004) conclude that a spatially weighted equivalent of the isolation index $I I_{g}$ and a spatially weighted equivalent of the information theory-based Shannon-Weaver index of diversity $S W$ are the optimal spatial segregation measures. Specifically, for a given individual at point $p$, let $\hat{\pi}_{p g}$ denote the average fraction of people of type $g$ in the neighborhood of point $p$, then

$$
\hat{E}_{p}=-\sum_{g=1}^{G} \hat{\pi}_{p g} \ln \left(\hat{\pi}_{p g}\right)
$$

defines the entropy of the local environment of $p$. The spatial information theory segregation index can then be defined by

$$
S S=1-\frac{1}{P_{\bullet .} S W} \int_{p \in R} \tau_{p} \widehat{E}_{p} d p
$$

in which $S W$ is the Shannon-Weaver diversity index of equation (5), $\tau_{p}$ is the population density at point $p, R$ indicates the entire region or city and the integral indicates that the index is calculated by summing over all points $p$. SS is a measure of how much less diverse the local environments of individuals are on average, compared with the diversity of the total population of region $R$. Further elaboration and a discussion of various other spatial indices can be found in Reardon and O’Sullivan (2004).

All measures discussed in this chapter thus far assume aggregation of individuals into groups with certain characteristics and into areas within certain boundaries. This aggregation approach was often the only feasible methodology in the past because data from population censuses or surveys were subject to strict confidentiality requirements to satisfy privacy legislation in many countries. Thus, published information on a population's demographic and socio-economic characteristics has been predominantly in the form of multi-way cross-tabulations with each cell reporting the observed number of people satisfying the specific classification (and often rounded to the base three to preserve confidentiality

\footnotetext{
${ }^{11}$ These properties are: (1) scale interpretability; (2) arbitrary boundary independence; (3) location equivalence; (4) population density invariance; (5) composition invariance; (6) transfers and exchanges; (7) additive spatial decomposability; and (8) additive grouping decomposability. See Reardon and O’Sullivan (2004) for details.
} 
requirements). However, researchers are now increasingly given access to micro data, with certain personal information (such as name and address) removed and replaced by a location indicator that is determined by the smallest spatial scale at which data are made available.

In recent years, many new sources of data are becoming available that provide very rich information, often combining survey and administrative data, at a very local level and sometimes even including the coordinates of the individual observation itself (particularly in GIS systems). Additionally, the information available from such integrated data infrastructures is often longitudinal: individual units are observed at various points in time. The emergence of this type of information calls for a new approach to measuring cultural diversity and segregation at the micro level. In this context, new operationalizations of socio-cultural and geographical distance are needed in the context of human interactions. For example, rather than measuring exposure to people from different backgrounds by means of simple shares of different socio-cultural groups in certain regions, network approaches can be used that account for face-to-face or virtual interaction between individuals. Alternatively, when location coordinates are known, the individual's activity space can be defined by spatial kernels (in which a group is defined by any individual captured within a certain radius of the individual) that can be varied in robustness checks. While the formal statistical analysis of spatial dependence and correlation remains computationally demanding for micro-level data, there are new promising developments (for an application to diversity among firms, see e.g. Dubé and Brunelle, 2014 and to multi-group spatial segregation of individuals, see e.g. Kumar et al., 2013). Parallel to the emergence of new rich micro data and conceptual developments, new statistical software for measurement of diversity and segregation is emerging too (e.g. Hong et al., 2014).

\section{$6 \quad$ CONCLUSION}

In this chapter we have argued that cultural diversity is emerging as an expanding and promising area of research in economics. Scientific inquiry into the socio-economic impacts of cultural diversity necessitates a thorough understanding of how to measure diversity in terms of differences between groups, or individuals, and differences between areas, or locations. In the past, economists have often adopted measures from 
a range of disciplines, such as ecology, biology, sociology, etc. While such measures can be easily calculated in any socio-economic application, their appropriateness is rarely critically assessed. In this context we reviewed in this chapter 29 different measures of diversity among people and places, which are among the most common in the literature. We have emphasized their interpretation and the relationships between them. We have not attempted a more formal mathematical or statistical evaluation of their properties, which can be found in e.g. Andreoli and Zoli (2012), Reardon and Firebaugh (2002), Reardon and O’Sullivan (2004) and White (1986).

Such evaluations show that for many measures the results are likely to depend on the extent of disaggregation of space and group attributes (i.e. the granularity of the data), the selection of relevant attributes and the population scale. An important warning in this context is that aggregate measures often suffer from the fallacy of composition. For example, as is clear from the literature on inequality, simply comparing countries in terms differences in their Gini coefficients provides very little information about the underlying income distribution and the often large differences across areas within countries. The same would be true for simply comparing cultural diversity across countries by a single index of fractionalization. It is therefore essential that sound micro, meso or macro conceptual frameworks and theories are adopted that provide guidance as to which cultural diversity measure is the most appropriate in the specific context.

For diversity among people, many papers have adopted the fractionalization index but it is clear that this captures just one aspect of cultural diversity and the concepts, and impacts, of other features of the data such as weighted abundance, entropy and polarization should also be considered, dependent on the context. For place diversity, researchers appear to have been most comfortable with measuring unevenness by means of the standard dissimilarity index. Here it is clear that measures of modified segregation, isolation, concentration, (cross) Moran's I and GIS-generated local measures such as location quotients, the local Moran and Getis and Ord's G* can provide complementing insights. In all of this, the definition of a 'group' is clearly moving away from a simple majority-minority dichotomy to the consideration of many groups defined by attributes across a range of domains.

The emerge of 'big data' and other exciting new data developments, such as integrated administrative and survey data infrastructures, suggest promising new approaches at the micro-level to measure cultural diversity in potential and actual 
contact/interaction between individuals. Much of the new data are temporal, and often longitudinal, which permits the testing of heterogeneity across cohorts, as well as life course approaches; aspects that have been neglected too much in the past given that most cultural diversity analyses to date have tended to be simply cross-sectional analyses. The longitudinal information also permits much more emphasis in the future on acculturation processes such as those that result from intermarriage and the absorption of the host country culture by first and subsequent generations of migrants.

Many studies that calculate diversity measures are purely descriptive in nature but there is still much scope for further multivariate (spatial) econometric models of the socio-economic impacts of cultural diversity. Varying the measures of diversity in such econometric models may inform on the aspects of diversity that matter most.

Ultimately, cultural diversity is in most applications an endogenous phenomenon. The endogeneity of constructed diversity measures is then as much of interest as their impacts, but accounting for reverse causality remains challenging. Randomized experiments of diversity impacts in economic contexts are rare (but see e.g. Boisjoly et al., 2006). Given the growing cross-border mobility and the associated emergence of super-diversity, we may expect a further widening and deepening of new research on the measurement of cultural diversity and its socio-economic impacts.

\section{References}

Alesina, A. and La Ferrara, E.L. (2005). Ethnic Diversity and Economic Performance. Journal of Economic Literature 43(3): 762-800.

Alesina, A., Devleeschauwer, A., Easterly, W., Kurlat, S. and Wacziarg, R. (2003). Fractionalization. Journal of Economic Growth 8: 155-194.

Alesina, A., Harnoss, J. and Rapoport, J. (2013). Birthplace Diversity and Economic Prosperity. NBER Working Paper No. 18699. Cambridge Mass.: National Bureau of Economic Research.

Alonso, W. (1964). Location and Land Use. Cambridge, MA: Harvard University Press.

Anderson, J.E. (2011). The Gravity Model. Annual Review of Economics 3: 133-160.

Andreoli, F. and Zoli, C. (2012). On the Measurement of Dissimilarity and Related Orders. ECINEQ Working Paper 2012-274. Verona, Italy: ECINEQ Society for the Study of Economic Inequality.

Anselin, L. (1988). Spatial Econometrics: Methods and Models. Dordrecht: Kluwer. 
Anselin, L. (1995). Local Indicators of Spatial Association - LISA. Geographical Analysis 27: 93-115.

Åslund, O. and Skans, N.O. (2009). How to Measure Segregation Conditional on the Distribution of Covariates. Journal of Population Economics 22: 971-981.

Audretsch, D., Dohse, D. and Niebuhr, A. (2010). Cultural Diversity and Entrepreneurship: A Regional Analysis for Germany. Annals of Regional Science 45: 55-85.

Bellini, E., Ottaviano, G., Pinelli, D. and Prarolo, G. (2008). Cultural Diversity and Economic Performance: Evidence from European Regions. HWWI Research Paper. Hamburg: Hamburg Institute of International Economics.

Benenson, I. (1998). Multi-agent Simulations of Residential Dynamics in the City. Computer, Environment and Urban Systems 22(1): 25-42.

Bishop, Y.M.M., Fienberg, S.E. and Holland, P.W. (1975). Discrete Multivariate Analysis: Theory and Practice. MIT Press.

Blaug, M. (2001). Where Are we Now on Cultural Economics? Journal of Economic Surveys 15(2): 123-143.

Boal, F.W. (1978). Ethnic Residential Segregation. In: D.T. Herbert and R.J. Johnston (eds.) Social Areas in Cities. London: Wiley.

Boisjoly, J., Duncan, G.J., Kremer, M., Levy, D.M. and Eccles, J. (2006). Empathy or Antipathy? The Impact of Diversity. American Economic Review 96(5): 1890-1905.

Borjas, G. (1998). To Ghetto or not to Ghetto: Ethnicity and Residential Segregation. Journal of Urban Economics 44: 228-253.

Bossert, W., D’Ambrosio, C. And La Ferrara, E. (2011). A Generalized Index of Fractionalization. Economica 78(312): 723-750.

Bossert, W., Pattanaik, P.K. and Xu, Y. (2003). Similarity of Options and the Measurement of Diversity. Journal of Theoretical Politics 15(4): 405-421.

Brock, W.A. and Durlauf, S.N. (2001). Discrete Choice with Social Interactions. Review of Economic Studies 68(2): 235-260.

Brown, L. and Chung, S.-Y. (2006). Spatial Segregation, Segregation Indices and the Geographical Perspective. Population, Space and Place 12(2): 125-143.

Bruch, E., and Mare, R. (2006). Neighborhood Choice and Neighborhood Change. American Journal of Sociology 112: 667-709.

Brunow, S., and Blien, U. (2014). Effects of Cultural Diversity on Individual Establishments. International Journal of Manpower, 35(1/2): 166-186.

Brunow, S., and Brenzel, H. (2012). The Effect of a Culturally Diverse Labour Supply on Regional Income in the EU, Empirica 39: 461-485.

Cliff, A., and Ord, J.K. (1981). Spatial Processes. London: Pion. 
Constant, A.F., and Zimmermann, K.F. (2008). Measuring Ethnic Identify and its Impact on Economic Behavior. Journal of the European Economic Association 6(2-3): 424-433.

Cutler, D. M., Glaeser, E.L., and Vigdor, J.L. (1999). The Rise and Decline of the American Ghetto. Journal of Political Economy 107: 455-506.

Desmet, K. Weber, S. and Ortuño-Ortín, I. (2009). Linquistic Diversity and Redistribution. Journal of the European Economic Association 7(6): 1291-1381.

Dubé, J. and Brunelle, C. (2014). Dots to Dots: A General Methodology to Build Local Indicators Using Spatial Micro-Data. Annals of Regional Science 53: 245-272.

Duncan, O.D. and Duncan, B. (1955). Residential distribution and occupational stratification. American Journal of Sociology, 60(5): 493-503.

Ellison, G. and Glaeser, E.L. (1997). Geographic Concentration in U.S. Manufacturing Industries: A Dartboard Approach. Journal of Political Economy 105: 889-927.

Esteban, J.M. and Ray, D. (1994). On the Measurement of Polarization. Econometrica 62(4): 819-851.

Esteban, J., Mayoral, L. and Ray, D. (2012). Ethnicity and Conflict: An Empirical Study. American Economic Review 102(4): 1310-1342.

Fearon, J.D. (2003). Ethnic and Cultural Diversity by Country. Journal of Economic Growth 8: 195-222.

Feinberg, W.E. (1985). Are Affirmative Action and Economic Growth Alternative Paths to Racial Equality? American Sociological Review 50(4): 561-571.

Fienberg, S.E. (2011). The Analysis of Contingency Tables: From Chi-Squared Tests and Log-Linear Models to Models of Mixed Membership. Statistics in Biopharmaceutical Research 3(2): 173-184.

Florax, R.J.G.M., de Graaff, T. and Waldorf, B.S. (2005). A Spatial Economic Perspective on Language Acquisition: Segregation, Networking, and Assimilation of Immigrants. Environment and Planning A 37: 1877-1897.

Fossett, M. (2006a). Ethnic Preferences, Social Distance Dynamics, and Residential Segregation: Theoretical Explorations Using Simulation Analysis, Journal of Mathematical Sociology, 30(3): 185-273.

Fossett, M. (2006b). Including Preference and Social Distance Dynamics in Multi-Factor Theories of Segregation, Journal of Mathematical Sociology 30(3): 289-298.

Friedrichs, J. (1998). Ethnic Segregation in Cologne, Germany, 1984-94. Urban Studies 35: 1745-1763.

Getis, A. and Ord, J.K. (1992). The Analysis of Spatial Association by Use of Distance Statistics. Geographical Analysis 24( 3): 189-206.

Gokhale, D.V. and Kullback, S. (1978). The Information in Contingency Tables. Marcel Dekker. 
Greenberg, J.H. (1956). The Measurement of Linguistic Diversity, Language 32: 109-115.

Gundelach, B. and Freitag, M. (2014). Neighbourhood Diversity and Social Trust: An Empirical Analysis of Interethnic Contact and Group-specific Effects. Urban Studies 51(6): 1236-1256.

Haas, A. and Lucht, M. (2013). Heterogeneous Firms and Imperfect Substitution: The Productivity Effect of Migrants. NORFACE MIGRATION Discussion Paper No. 201319, www.norface-migration.org

Heckman, J.J., Lochner, L. and Taber, C. (1998). Explaining Rising Wage Inequality: Explanations With A Dynamic General Equilibrium Model of Labor Earnings With Heterogeneous Agents. Review of Economic Dynamics 1(1): 1-58.

Hofstede, G. (2001). Culture’s Consequences. Second edition. Sage Publications.

Hong, S-Y, O’Sullivan, D. and Sadahiro, Y. (2014). Seg: Implementing Recent Developments in the Measurement of Segregation. Center for Spatial Information Science, University of Tokyo.

Ihlanfelt, K., and Scafidi, B. (2002). Black Self Segregation as a Cause of Housing Segregation: Evidence from the Multi-City of Urban Inequality, Journal of Urban Economics 51: 366-399.

Ioannides, Y.M. (2010). A Review of Scott E. Page's The Difference: How the Power of Diversity Creates Better Groups, Firms, Schools, and Societies. Journal of Economic Literature 48(1): 108-122.

Johnston, R., Poulsen, M. and Forrest, J. (2009). Research Note-Measuring Ethnic Residential Segregation: Putting Some More Geography In. Urban Geography 30(1): 91109.

Johnston, R.J., Poulsen, M. and Forrest, J. (2007). The Geography of Ethnic Residential Segregation: A Comparative Study of Five Countries. Annals of the Association of American Geographers 97: 713-738.

Karakayali, N. (2009). Social Distance and Affective Orientations. Sociological Forum 23(3): 538-562.

Kloosterman, R.C. and Rath, J. (2001). Immigrants Entrepreneurs in Advanced Economies. Journal of Ethnic and Migration Studies 27(2): 189-201.

Knotter, S., de Lobel, R., Tsipouri, L. and Stenius, V. (eds.) (2011) Diversity Research and Policy. Amsterdam: Pallas.

Kumar, N., White, M.J., and Singh, G. (2013) Multi-group Individual Level Measures of Segregation. Available at SSRN: http://ssrn.com/abstract=2205714.

Longhi, S. (2014). Cultural Diversity and Subjective Wellbeing. IZA Journal of Migration 3: 13. 
Maignan, C., Ottaviano, G., Pinelli, D. and Rullani, F. (2003). Bio-Ecological Diversity vs. Socio-Economic Diversity: A Comparison of Existing Measures. Working Paper 13.2003, Fondazione Eni Enrico Mattei, Milan.

Maré, D.C., Pinkerton, R.M., Poot, J. and Coleman, A. (2012). Residential Sorting Across Auckland Neighbourhoods. New Zealand Population Review 38: 23-54.

Margalef, D.R. (1958). Information Theory in Ecology. General Systematics 3:36-71.

Massey, D.S. and Denton, N.A. (1988). Dimensions of Residential Segregation. Social Forces 67(2): 281-315.

Maurel, F. and Sédillot, B. (1999). A Measure of the Geographic Concentration in French Manufacturing Industries. Regional Science and Urban Economics 29: 575-604.

McAfee, A. and Brynjolfsson, E. (2012). Big Data: The Management Revolution. Harvard Business Review, October.

McMahon, A. and McMahon, R. (2005). Language Classification by Numbers. Oxford: Oxford University Press.

Moretti, E. (2011). Local Labor Markets. In: Handbook of Labor Economics. Volume 4, Part B, pp. 1237-1313.

Moro, A., and P. Norman, P. (2003). Affirmative Action in a Competitive Economy. Journal of Public Economics 87: 567-594.

Musterd, S. and van Kempen, R. (2009). Segregation and Housing of Minority Ethnic Groups in Western European Cities. Tijdschrift voor Economische en Sociale Geografie, 100: 559-566.

Muth, R. (1971). The Derived Demand for Urban Residential Land, Urban Studies 8(2): 243254.

Olfert, M.R., and M. Partridge, M. (2011). Creating the Cultural Community: Ethnic Diversity vs. Agglomeration. Spatial Economic Analysis 6(1): 25-35.

Ottaviano, G. and Peri, G. (2006). The Economic Value of Cultural Diversity: Evidence from US Cities. Journal of Economic Geography 6(1): 9-44.

Ozgen, C., Nijkamp, P. and Poot, J. (2010). The Effect of Migration on Income Growth and Convergence: Meta-Analytic Evidence. Papers in Regional Science 89(3): 537-561.

Ozgen, C., Nijkamp, P. and Poot, J. (2012). Immigration and Innovation in European Regions. In: P. Nijkamp, J. Poot and M. Sahin (eds.) Migration Impact Assessment: New Horizons Cheltenham: Edward Elgar.

Ozgen, C., Nijkamp, P. and Poot, J. (2013). The Impact of Cultural Diversity on Innovation: Evidence from Dutch Firm-Level Data. IZA Journal of Migration 2:18.

Ozgen, C., Peters, C., Niebuhr, A., Nijkamp, P. and Poot, J. (2014). Does Cultural Diversity of Migrant Employees Affect Innovation? International Migration Review 48(S1): S377S416. 
Page, S.E. (2007). The Difference: How the Power of Diversity Creates Better Groups, Firms, Schools, and Societies. Princeton: Princeton University Press.

Patil, G.P. and Taillie, C. (1982). Diversity as a Concept and its Measurement. Journal of the American Statistical Association 77(379): 548-561.

Pawlak, Z. (1997). Rough Set Approach to Knowledge-Based Decision Support. European Journal of Operational Research 99(1): 48-57.

Phillips, D. (1998). Black Minority Ethnic Concentration, Segregation and Dispersal in Britain. Urban Studies 35: 1681-1702.

Pisatio, M. (2001). Tools for spatial data analysis. Stata Technical Bulletin, STB-60, 12-36.

Poot, J., Waldorf, B. and van Wissen, L. (eds) (2008). Migration and Human Capital. Edward Elgar, Cheltenham UK.

Putnam, R.D. (2000). Bowling Alone. New York: Simon \& Schuster.

Putnam, R.D. (2006). E Pluribus Unum. Scandinavian Political Studies 30: 137-174.

Ratna, N.N., Grafton R.Q. and T. Kompas (2009). Is Diversity Bad for Economic Growth? Evidence from State-level Data in the US. Journal of Socio-Economics 38: 859-870.

Reardon, S.F. and Firebaugh, G. (2002). Measures of Multigroup Segregation. Sociological Methodology 32(1): 33-67.

Reardon, S.F. and O’Sullivan, D.O. (2004). Measures of Spatial Segregation. Sociological Methodology 34(1): 121-162.

Reynal-Querol, M. (2002). Ethnicity, Political Systems and Civil Wars. Journal of Conflict Resolution 46(1): 29-54.

Roback, J. (1982). Wages, Rents, and the Quality of Life. Journal of Political Economy 90(6): 1257-1278.

Rousseau, R., Van Hecke, P., Nijssen, D. and Bogaert, J. (1999). The Relationship between Diversity Profiles, Evenness and Species Richness based on Partial Ordering. Environmental and Ecological Statistics 6: 211-223.

Sahin, M., Nijkamp, P. and Stough, R. (2010). Impact of Urban Conditions on Firm Performance of Migrant Entrepreneurs. The Annals of Regional Science 46(3): 661-689.

Sakoda, J.M. (1971). The Checkerboard Model of Social Interaction. Journal of Mathematical Sociology 1: 119-132.

Schelling, T.C. (1971). Dynamic Models of Segregation. Journal of Mathematical Sociology 1: $143-186$.

Shenkar, O. (2001). Cultural Distance Revisited: Towards a More Rigorous Conceptualization and Measurement of Cultural Differences. Journal of International Business Studies 32(3): 519-535.

Speare, A. (1974). Residential Satisfaction as an Intervening Variable in Residential Mobility, Demography 11: 173-188. 
Sturgis, P., Brunton-Smith, I., Kuha, J. and Jackson, J. (2014). Ethnic diversity, segregation and the social cohesion of neighbourhoods in London. Ethnic and Racial Studies 37(8): 1286-1309.

Suedekum, J., Wolf, K. and Blien, U. (2014). Cultural Diversity and Local Labour Markets. Regional Studies 49(1): 173-191.

Theil, H. (1972). Statistical Decomposition Analysis. Amsterdam: North-Holland.

Theil, H. and Finezza, A.J. (1971). A Note on the Measurement of Racial Integrtaion of Schools by Means of Informational Concepts. Journal of Mathematical Sociology 1: 187194.

Trax, M., Bruno, S. and J. Suedekum, J. (2012). Cultural Diversity and Plant Level Productivity. NORFACE MIGRATION Discussion Paper No. 2012-29, www.norfacemigration.org

van Kempen, R. (2005). Segregation and Housing Conditions of Immigrants in Western European Cities. In: R. Kazepov (ed.) Cities of Europe, Changing Contexts, Local Arrangements, and the Challenge to Urban Cohesion. Oxford: Blackwell.

van Mourik, A., Poot, J. and Siegers, J. J. (1989). Trends in Occupational Segregation of Women and Men in New Zealand. New Zealand Economic Papers 23: 29-50.

Vertovec, S. (2007). Super-diversity and its implications. Ethnic and Racial Studies 30(6): 1024-1054.

White, M.T. (1986). Segregation and Diversity Measures in Population Distribution. Population Index 52(2): 198-221.

Whittaker R.H. (1977). Evolution of Species Diversity in Land Communities. Evolutionary Biology 10: 1-67.

Wong, D.W.S. and Shaw, S.-L. (2011). Measuring Segregation: An Activity Space Approach. Journal of Geographical Systems 13(2): 127-146. 


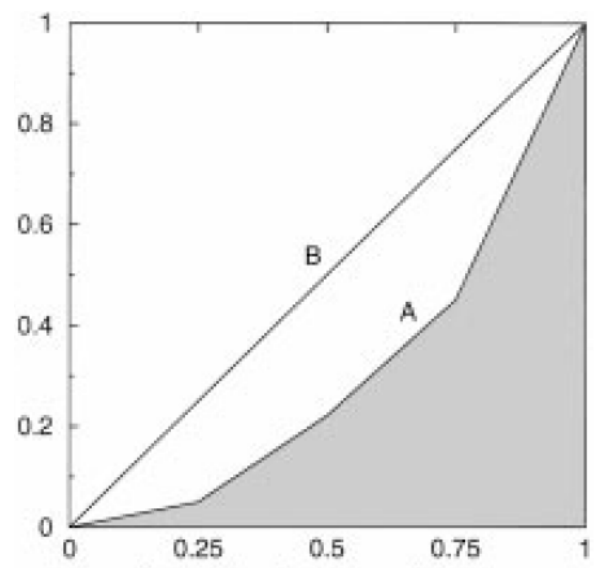

Cumulative Proportion of Groups

Figure 1 The Gini index and Lorenz curve

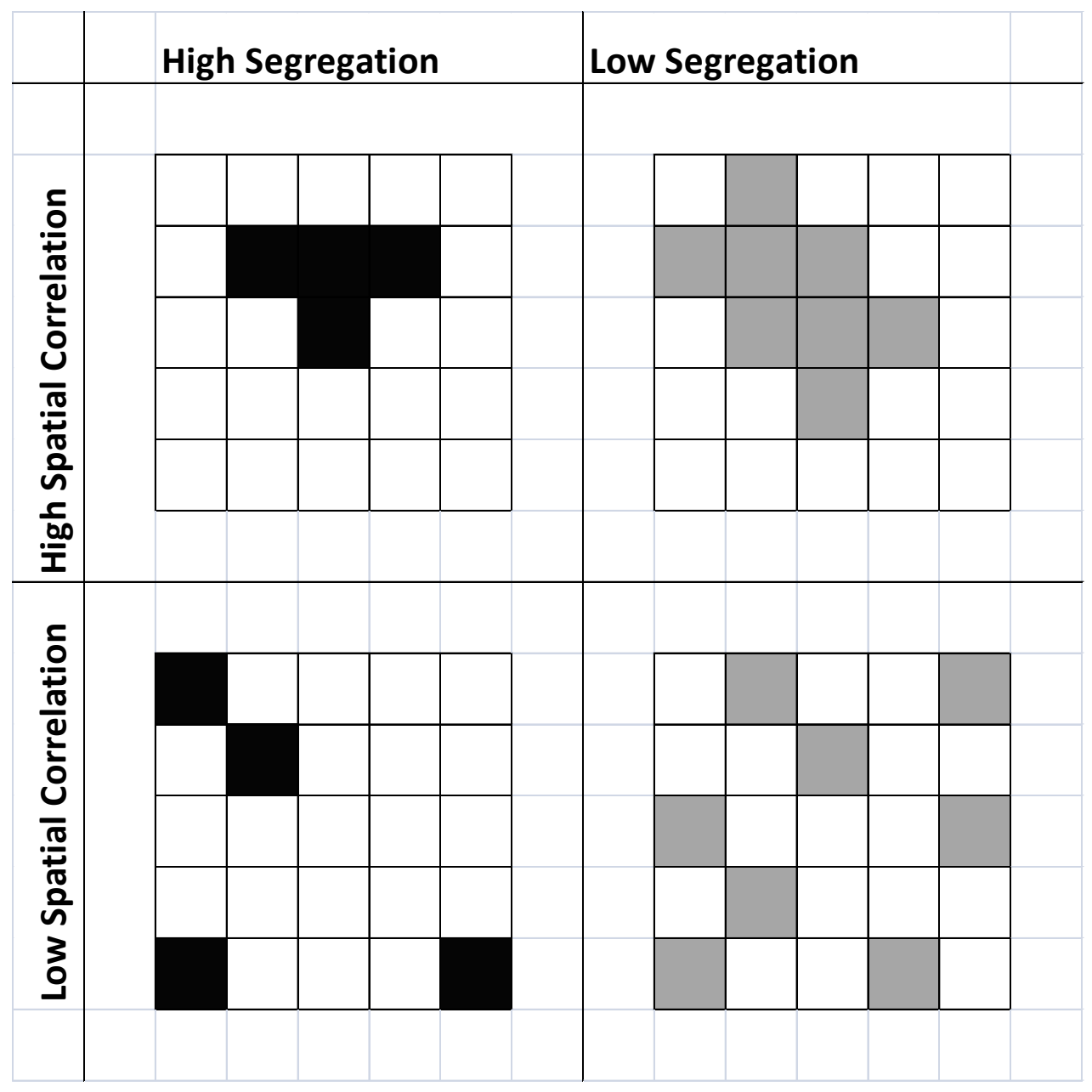

Figure 2 Comparing segregation and spatial correlation 


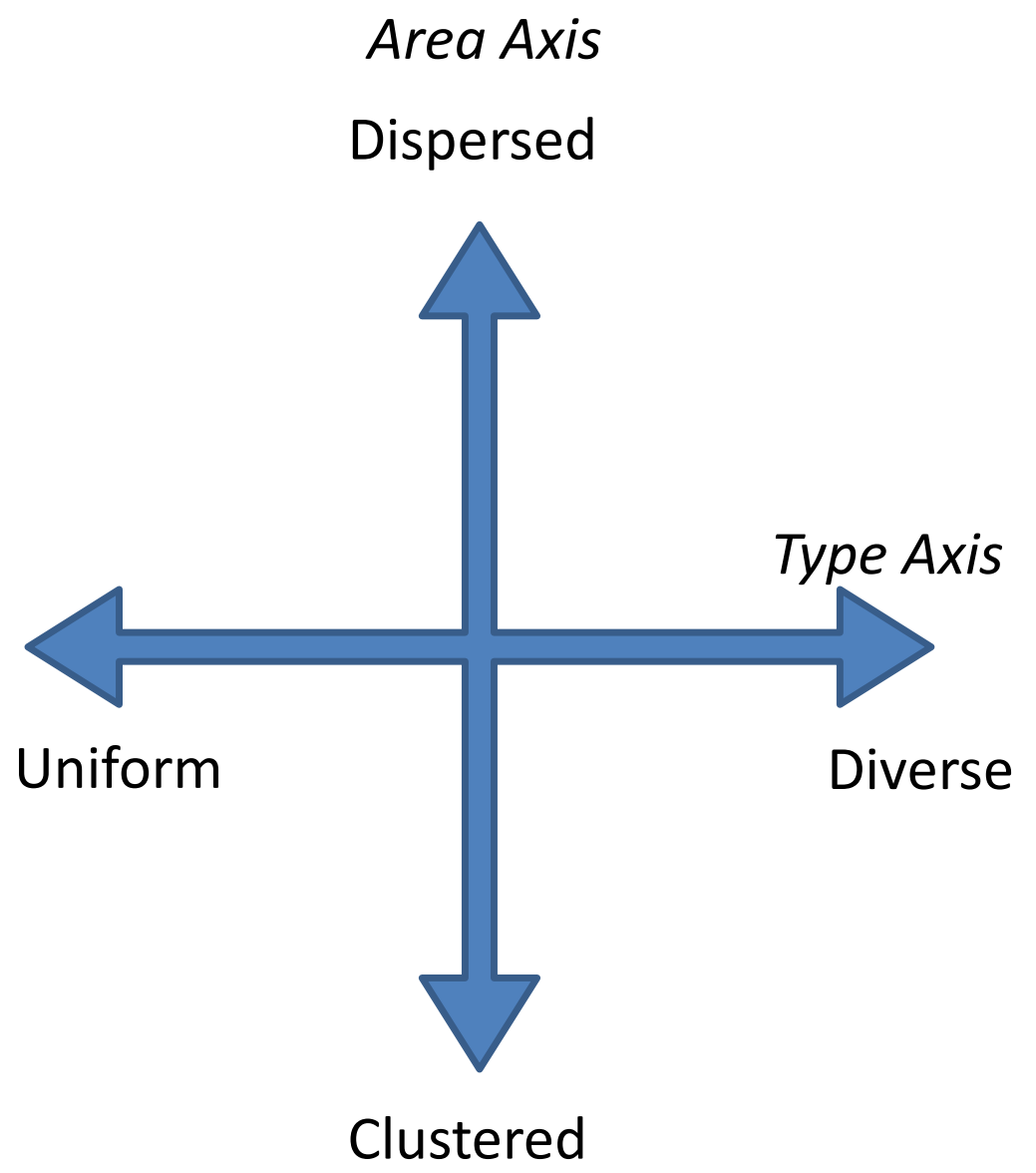

Figure 3 Combining Area and Group Diversity 